\title{
Performance of the Pixel Luminosity Telescope for Luminosity Measurement at CMS during Run 2
}

\author{
Paul Lujan* (Universita e INFN, Padova) on behalf of the CMS Collaboration \\ E-mail: paul.lujan@cern.ch
}

The Pixel Luminosity Telescope (PLT) is a dedicated system for luminosity measurement at the CMS experiment using silicon pixel sensors arranged into "telescopes", each consisting of three sensor planes. It was installed in CMS at the beginning of 2015 and has been providing online and offline luminosity measurements throughout Run 2 of the LHC. The online bunch-by-bunch luminosity measurement employs the "fast-or" capability of the pixel readout chip to identify events where a hit is registered in all three sensors in a telescope, corresponding primarily to tracks originating from the interaction point. In addition, the full pixel information is read out at a lower rate, allowing for the calculation of corrections to the online luminosity from effects such as the miscounting of tracks not originating from the interaction point and detector efficiency. This paper presents results from the 2016 running of the PLT, including commissioning and operational history, luminosity calibration using Van der Meer scans, and corrections to the online luminosity, as well as offline performance and monitoring.

The European Physical Society Conference on High Energy Physics

5-12 July

Venice, Italy

\footnotetext{
* Speaker.
} 


\section{Introduction}

The Pixel Luminosity Telescope (PLT) is a dedicated luminosity measurement system which was installed in the CMS detector [1] at the beginning of 2015 for operations during Run 2 of the LHC. The telescope consists of 48 silicon pixel sensors arranged into 16 "telescopes", where each telescope consists of three silicon sensor planes mounted near the beampipe perpendicular to the beam direction separated by a total of approximately $7.5 \mathrm{~cm}$, such that a track coming from the interaction point (IP) passes through all three sensors in a telescope. The full PLT consists of eight telescopes mounted on each side of CMS, outside the pixel endcap, at a $|\eta|$ of approximately 4.2.

The PLT uses the same silicon sensors [2] and readout chips (ROCs) [3] as in the phase-0 CMS pixel detector. However, the PLT takes advantage of a feature in the PSI46v2 ROC not used in the main pixel detector: the "fast-or" readout, which reads out a signal indicating if any of the pixels in the sensor were hit at the full bunch crossing rate of $40 \mathrm{MHz}$, thus allowing the PLT to provide online per-bunch luminosity with excellent statistical precision on a very short timescale. The luminosity measurement using the fast-or readout looks for "triple coincidences", where we observe a hit in all three planes in a single telescope; the rate of triple coincidences should be proportional to the luminosity. The full pixel data can also be read out at a lower rate (approximately $3.3 \mathrm{kHz}$ during 2016-2017) for additional studies.

The three ROCs in a telescope are controlled by a Token Bit Manager (TBM) chip [4], which distributes commands to and coordinates the readout of the individual ROCs. Four telescopes make up a "quadrant" of the PLT, and are controlled by a single "port card", which manages the communication and control signals for that quadrant. The port card is in turn connected to an optical motherboard, which translates the electrical signals into optical signals which are then transmitted via optical fibers from the CMS experimental cavern into the CMS service cavern where the backend electronics are located.

The back-end electronics consist of three parts: the Front-End Controller (FEC) is responsible for sending commands, as well as clock and trigger signals, to the detector; the pixel Front-End Driver (FED) reads out and decodes the pixel data, which is then sent over an Slink connection to a dedicated PC; and the fast-or FEDs look for triple coincidences and histogram the results over a "lumi nibble" (4096 orbits), which are then read out over a CAEN VME optical bridge to a dedicated PC. From there the data is sent to BRILDAQ, a separate DAQ system dedicated to luminosity and beam measurements. BRILDAQ operates separately from the main CMS DAQ so that it can continue to provide luminosity measurements to the LHC and beam safety measurements even when the CMS DAQ is not running.

The sensors consist of 80 rows and 52 columns of pixels, where each pixel is $100 \mu \mathrm{m}$ by $150 \mu \mathrm{m}$, for a total active area of $8 \times 8 \mathrm{~mm}$. However, to decrease the contribution from accidentals (discussed further in Sec. 3), only a smaller active area of the sensor is used. In 2015, the active area was $4.2 \times 4.0 \mathrm{~mm}$ ( 28 columns by 40 rows) in the central plane of a telescope and $5.4 \times 5.2 \mathrm{~mm}$ ( 36 columns by 52 rows) in the outer planes, while in 2016 the active area was further decreased to $3.6 \times 3.6 \mathrm{~mm}$ ( 24 columns by 36 rows) in the center plane and $3.9 \times 3.8 \mathrm{~mm}$ ( 26 columns by 38 rows) in the outer planes.

In 2015, two of the telescopes stopped working due to a failure of the low-current differential signal (LCDS) chips on the port card which communicate with the TBM. The port card was re- 
placed during the extended year-end technical stop (EYETS) in 2016-2017 and the two telescopes were returned to operation. Two more telescopes suffered failures in 2016 where the pixel readout stopped working, although the fast-or readout was unaffected. These were not repairable within the EYETS timescale, so for 2017 they are excluded from the luminosity calculation.

\section{Luminosity Measurement and Calibration}

In principle, the average rate of triple coincidences $\mu$ measured in the PLT should be proportional to the instantaneous luminosity delivered by the LHC. However, because of the limitations of the fast-or algorithm used in the ROCs, it is possible that multiple particle tracks could be registered as only a single triple coincidence in a telescope. Thus, in order to avoid the possibility of error from this effect, we instead use the "zero-counting" technique: if $f_{0}$ is the fraction of events with no triple coincidence measured, then $\mu=-\ln f_{0}$. The $\mu$ value is then related to the instantaneous luminosity by the equation

$$
\frac{d L}{d t}=\frac{\mu f_{\text {orb }}}{\sigma_{\text {vis }}}
$$

where $f_{\text {orb }}$ is the LHC orbit frequency of $11246 \mathrm{~Hz}$ and $\sigma_{\text {vis }}$ is the "visible cross section", the fraction of the total inelastic cross section which is visible to the PLT. To determine the overall luminosity, $\mu$ is measured bunch-by-bunch and telescope-by-telescope; for a single bunch, $\mu$ is averaged over all telescopes, and then converted to the instantaneous luminosity. The total instantaneous luminosity is then given by the sum over all bunches.

To determine $\sigma_{\mathrm{vis}}$, we perform a calibration using the Van der Meer (VdM) scan method [5]. This method relies on the fact that the instantaneous luminosity is given as a function of the beam parameters:

$$
\frac{d L}{d t}=\frac{N_{1} N_{2} f_{\text {orb }}}{2 \pi \Sigma_{x} \Sigma_{y}}
$$

where $N_{1}$ and $N_{2}$ are the LHC beam intensities and $\Sigma_{x}$ and $\Sigma_{y}$ are the effective beam widths. (In the case where the beams can be taken to be Gaussian, $\Sigma$ is simply equal to the $\sigma$ of the Gaussian distribution.)

The VdM scan is performed by varying the beam separation between $-6 \sigma$ and $+6 \sigma$ in steps of $0.5 \sigma$, first in the $x$ plane and then in the $y$ plane, and measuring the resulting rate as a function of separation. The rate curve is then fit with a function consisting of two Gaussian terms and a constant term. The $\Sigma$ values are thus extracted from the fits, and given the measured beam currents from the LHC, the absolute luminosity can then be calculated, and thus the calibration constant relating the measured $\mu$ in the PLT to the absolute luminosity is defined. Figure 1 shows the fit procedure for a single bunch, as well as the results across all bunches for all five scan pairs in the 2016 VdM program. We observe consistent results across all bunches and scans.

\section{Systematic Effects and Corrections}

In this section we discuss various effects which affect the accuracy of the PLT luminosity measurement and how we correct for them. 

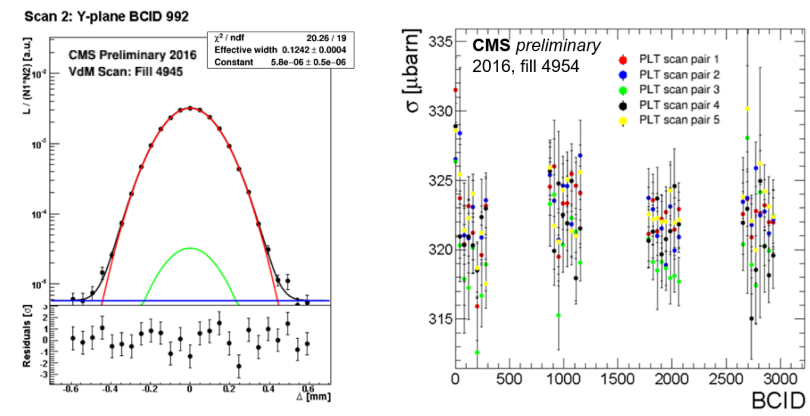

Figure 1: Left: Measured rate in the PLT as a function of the beam separation in a VdM scan for a single bunch crossing ID (BCID). The components of the fit are shown by the different colors: two Gaussian terms (red and green) and a constant term (blue). Right: Measured $\sigma_{\mathrm{vis}}$ as a function of the bunch crossing ID for the five scan pairs in the $2016 \mathrm{VdM}$ program.

\subsection{Accidentals}

The principal source of systematic uncertainty in the PLT measurement is "accidentals"; that is, events where we observe a triple coincidence in the fast-or measurement that does not originate from a genuine track from the IP. This can be due either to a track from another source (e.g. a "beam halo" particle passing through the PLT) or due to a random combination of hits not from a single track at all (from cosmic rays, radioactive decay of activated material, secondary material interactions in the detector, and so on). Because the fast-or data does not include position information, these cannot be identified in the fast-or data, but we can use the full pixel data to estimate the rate at which they occur in the fast-or data and develop corrections.

The primary accidental measurement used in 2016 reconstructs tracks from a "pure" sample of events where each plane in the telescope has exactly one hit. The distributions of the track slopes and residuals on each plane are then computed and used as a reference. A candidate track is then considered to be "accidental" if any of the slopes or residuals is more than $5 \sigma$ away from the mean of the reference distribution. In the case where there are multiple candidate tracks in a telescope, the event is considered good if any possible combination is good, because of the zero-counting technique.

Figure 2 shows the resulting measured accidental rate as a function of the instantaneous luminosity over the course of several fills. We observe that the accidental rate is roughly linear with the instantaneous luminosity, and is relatively consistent over these fills. However looking over full 2016 data set, we observe that the measured slope of the accidental rate decreases over the course of 2016. These measurements are used to derive a final correction for the accidentals in the PLT luminosity.

\subsection{Efficiency}

In mid-2016, we observed that the ratio of the PLT luminosity to the luminosity measured by other CMS luminometers declined during periods of high-intensity running, and then recovered after a technical stop. This was found to be due to the effect of radiation damage in the sensors being greater than expected; in October, the high voltage applied to the sensors was raised from 

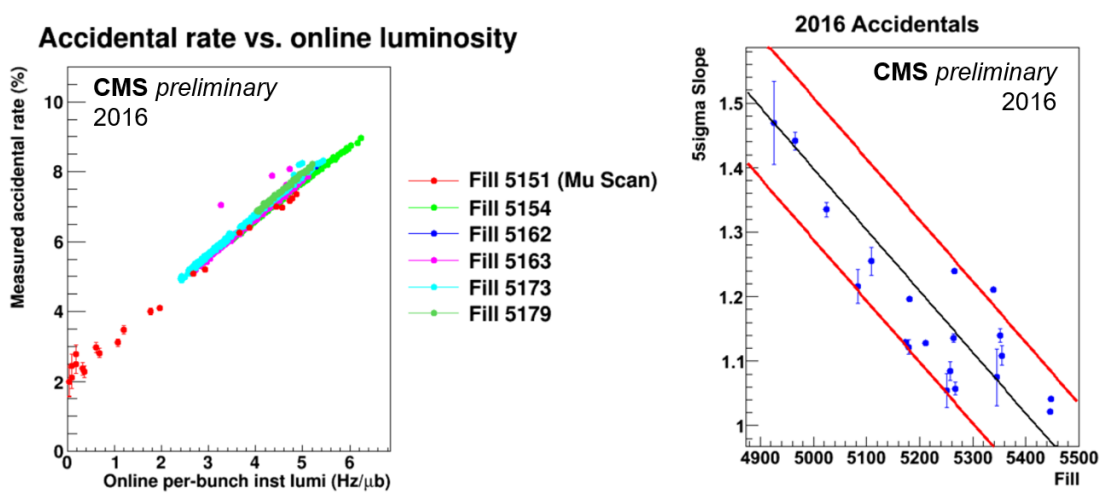

Figure 2: Left: Accidental rate vs. instantaneous luminosity for a set of fills. Fill 5151 is a special "mu scan" fill where the beams were separated during normal physics data taking to allow for measurements over a wider range of luminosity. Right: Measured slope of accidental rate vs. instantaneous luminosity over the course of the 2016 run. A downward trend is observed.

$150 \mathrm{~V}$ to $200 \mathrm{~V}$, which worked to mitigate further loss. In order to correct the measurement during the affected periods, we measured the efficiency in the PLT by looking for events with two hits in two planes consistent with a track passing through the third plane, and then measuring the fraction the third hit was actually found in the third plane. This was used to derive a correction for the PLT efficiency loss during 2016. Figure 3 shows the results of this correction.
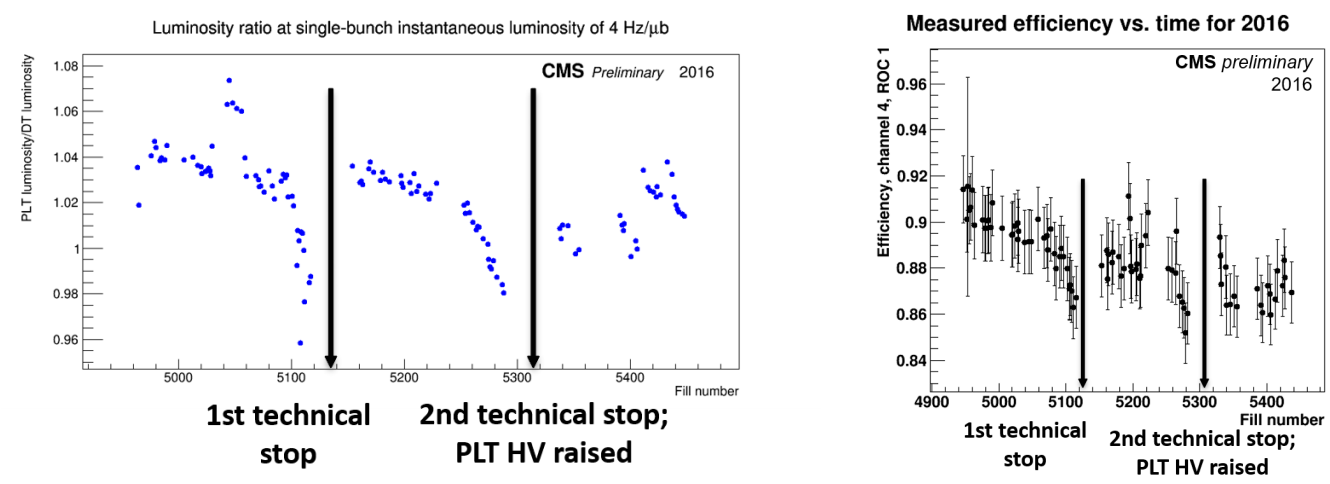

Figure 3: Left: Ratio of PLT to DT luminosity over the course of 2016. The two periods of PLT efficiency loss are clearly visible. Right: Measured efficiency in the PLT over the course of 2016. This measurement is used to derive a correction to the measured PLT luminosity.

\section{Summary}

The Pixel Luminosity Telescope (PLT) has been successfully delivering luminosity measurements to CMS and LHC since the beginning of 2015 with high uptime and precision. Some operational issues in 2016 were successfully corrected with later analysis. We look forward to successful operation of the PLT during 2017 running and beyond. 


\section{References}

[1] CMS collaboration, S. Chatrchyan et al., The CMS Experiment at the CERN LHC, JINST 3 (2008) S08004.

[2] Y. Allkofer et al., Design and performance of the silicon sensors for the CMS barrel pixel detector, Nucl. Instrum. Meth. A584 (2008) 25-41, [physics / 0702092 ].

[3] H. C. Kästli, M. Barbero, W. Erdmann, C. Hormann, R. Horisberger, D. Kotlinski et al., Design and performance of the CMS pixel detector readout chip, Nucl. Instrum. Meth. A565 (2006) 188-194, [physics/0511166].

[4] E. Bartz, The 0.25 $\mu \mathrm{m}$ token bit manager chip for the CMS pixel readout, in $11^{\text {th }}$ Workshop on Electronics for LHC and Future Experiments, CERN, 2005, DOI.

[5] S. van der Meer, Calibration of the effective beam height in the ISR, Tech. Rep. CERN-ISR-PO-68-31. ISR-PO-68-31, CERN, Geneva, 1968. 